did not attend \& increased healthcare utilisation post-referral. Patient 106 had increased healthcare utilisation post-referral from a new health condition. The randomised sample identified limitations of using healthcare utilisation as an outcome measure when contrasted to the non-randomised case (which significantly reduced healthcare utilisation post-referral).

Conclusion. Correlation only can be inferred from the data due to sample size, limitations \& confounding factors e.g. psycho-social life events, acquired illness. Alternative outcome measurements documented (e.g PHQ9/GAD7) were not reliably recorded across pathways.

The results evidenced that single cases can demonstrate highly desirable effects of a biopsychosocial approach but they can also skew data sets if results are pooled due to the small sample size \& heterogeneous interventions. With some patients an increase in healthcare utilisation was appropriate for an improved clinical outcome. This audit identified that utilising healthcare utilisation as an outcome measure is a crude tool with significant limitations $\&$ the need to agree tailored outcome measures based on the type of intervention to assess the impact of IPMS.

\section{Reasonable adjustments for autistic adults}

Nazish Hashmi* and Conor Davidson

Leeds and York NHS foundation trust

${ }^{*}$ Corresponding author.

doi: 10.1192/bjo.2021.253

Aims. To embed the use of reasonable adjustments for adults with autism within in mental health services.

Objectives. The objectives of the project are as follows:

To identify how many service users with a diagnosis of autism are under care of local mental health services

Is there evidence that reasonable adjustments were considered for these service users

If identified as needing reasonable adjusments is there evidence of such adjustments being made

Method. We looked at service users with an established diagnosis of autism under care of Leeds and York NHS foundation trust to ascertain if reasonable adjustments have been considered. The audit is based on guidelines provided by Think Autism-department of health statutory guidance 2014. This is based on autism act 2009.

Data were collected for 30 cases in mainstream mental health services undr care of various teams including inpatient and community.

Result. It was identified that in only $2 / 30$ cases reasonable adjustments were considered and agreed upon. Only 1/30 service users had a disability status updated on electronic patient records. None of the service users had a hospital passport or reasonable adjustment care plan completed.None of the records had "good evidence" of reasonable adjustments.

These findings point to a wider issue for the trust as well as natioanlly as it indicates that autism is not being adequately taken into account for patients accessing our services. Due to the lack of reasonable adjustments adults with autism are potentially at increased risk to disengage leading to deterioration in their mental state and increase in risks.

Conclusion. These findings point to a wider issue for the trust as it indicates that autism is not being adequately taken into account for patients accessing our services. Due to the lack of reasonable adjustments adults with autism are potentially at increased risk to disengage leading to deterioration in their mental state and increase in risks.

We recommend training in autism for all healthcare professionals in the trust to improve their understanding of autism, including making reasonable adjustments.

We also recommend review trust procedure about recording diagnoses and disability status on electronic patient records. We recommend that the reasonable adjustments section on care director is more prominent and easily accessible.

We recommend that an 'autism flag' is prominent on patient records to alert staff to the presence of autism

\section{Dementia screening: an audit of screening for reversible causes of dementia}

Kim Herbert ${ }^{1 \star}$, Elspeth Richardson ${ }^{2}$, Adam Daly ${ }^{1}$

and Christine Carswell ${ }^{1}$

${ }^{1}$ NHS Lanarkshire and ${ }^{2}$ NHS Greater Glasgow and Clyde

${ }^{*}$ Corresponding author.

doi: $10.1192 /$ bjo.2021.254

Aims. This audit aimed to assess to what extent patients being referred for assessment of memory problems were receiving appropriate screening for reversible causes. We considered the blood tests recommended by the National Institute for Clinical Excellence (NICE).

Background. Research into 'reversible dementias' identified numerous common underlying causes. As a result of this NICE complied comprehensive guidance on investigations which should be performed in the initial stages of assessing patients with memory problems, ideally at a primary care level. These investigations are also crucial at the point of secondary care assessment in order to make a confident diagnosis.

Method. Details of patients referred by their GP to the Older Adult CMHT with memory problems over a one month period were collected. We then used the local laboratory database to note whether each of the eight recommended blood tests had been performed in the preceding 6 months. We measured this against an agreed standard of $95 \%$.

After the first cycle of data collection we prepared businesscard sized 'aide memoirs' for GPs that could serve as a quick reminder. These were sent out to all GPs in the area along with a letter outlining the audit findings.

Result. Overall 31 patients were included in the first cycle. 15 patients had all 8 dementia blood screens (48\%), 13 (42\%) had some of the recommended tests and 3 patients had no screening tests at all (10\%). On average patients had $76.6 \%$ of the recommended bloods completed. The most commonly completed tests were Full Blood Count (FBC) and Urea \& Electrolytes (U\&Es), with blood Glucose being the most frequently omitted.

In cycle 2, 20 patients were included. Of these patients, 10 had the full complement of screening bloods (50\%); 8 had some tests completed (40\%) and 2 patients had no screening tests complete $(10 \%)$. On average $76 \%$ of tests were completed. There was an improvement in the rate of completion of both Glucose and Liver Function Tests from cycle1.

Conclusion. This audit demonstrated that current practice does not meet the national standard in general. Our intervention produced a modest improvement in the proportion of patients who received a full complement of dementia screening tests, as well as increasing the rate of patients receiving a blood glucose as part of their screening. It would likely be beneficial to consider further intervention and a 3 rd audit cycle in due course. 